Letras, Lima 48 (82-83): 55-62, 1976.

\title{
En torno al problema del tiempo
}

ARSENIO GUZMAN JORQUERA

En el presente estudio circunscribimos el análisis de ciertos aspectos del problema del tiempo al mundo físico. Consideramos que es en este plano en donde cualquier tratamiento objetivo de los problemas relacionados con el tiempo tiene que desarrollarse. En consecuencia, sostenemos que los diversos juicios que, saturados de matices emotivos, sobre el llamado tiempo subjetivo se emiten son irrelevantes, en general, en lo tocante al esclarecimiento de lo que sea el tiempo y de las propiedades que se le atribuyen. No negamos, por lo demás, el valor que en otros terrenos, el de la poesía por ejemplo, semejantes juicios pudieran tener.

Aunque la observación del mundo que nos rodea parece proporcionar un sólido fundamento para Admitir la presencia de algo que figuradamente podemos denominar "flujo temporal", no creemos, sin embargo, que haya base suficiente para aceptar, en sentido estricto, un efectivo darse de tal "flujo", por lo menos no en el sentido de un "puro fluir" en el cual tengan lugar las cosas. Naturalmente, cualquier observador puede informarnos sobre cambios ocurridos en las cosas y su informe será sustancialmente correcto, pero interpretar esto como un acontecer de las cosas en el tiempo (o como un participar de su fluir) no parece ser igualmente correcto.

Concebimos el mundo real como un conjunto de procesos (1) y admitimos que el desarrollo implicado en todo proceso es temporal. No obstante, el reconocer tal situación no significa que podamos o debamos examinar separadamente procesos y temporalidad, caso en el cual inevitablemente surgirían dificultades, como

(1) En el sentido físico de la palabra, puede entenderse procesos como transformaciones energéticas. Este es el uso que en general hacemos del término. En tal sentido, si en los procesos tenemos concatenaciones de acaecimientos, es la energía la que lleva de un acaecimiento a otro. 
posteriormente podrá verse; sino que, por el contrario, resulta mucho más adecuado a los hechos, y más conveniente para la resolución o eliminación de numerosos problemas, admitir que, bien vistas las cosas, procesos y temporalidad se encuentran unidos, en el sentido de darse la sucesión temporal como un modo de ser de los procesos. Esto quiere decir que no es el caso de que el proceso sea posible únicamente si le precede la temporalidad como condición. Consideramos más apropiado sostener que fuera de los procesos, esto es, sin la existencia de ellos, carece de sentido hablar de una temporalidad. Por supuesto, es enteramente posible pensar en la temporalidad en sí misma, y en los procesos como algo que tiene lugar en el tiempo, en el cual y por el cual éstos son temporales; pero hacer esto significaría, en el primer caso, proceder exclusivamente en el nivel de la pura abstracción $y$, en el segundo, una inadecuada manera de enfocar la situación. El proceso es temporal, mas no significa esto que el proceso está en el tiempo. El tiempo no debe entenderse sino como concepto que designa la temporalidad dada en el proceso, como una nota constitutiva de éste, y no hay temporalidad sin proceso. Por lo demás, si tiempo significa temporalidad, entonces vale decir que hay tiempo sólo porque hay procesos.

Naturalmente, lo dicho nos lleva a pensar en la necesidad de una pequeña, pero importante, aclaración respecto de lo que habitualmente se entiende por proceso. No deberá éste ser considerado como una serie o concatenación de acaecimientos ordenada en el tiempo (2), puesto que tiempo es tan sólo un término que refiere a una nota constitutiva del proceso. Pensar en procesos como series de "đggaecimientosebrdenadossen el tiempo llevaría a presuponer un tiempo independiente de todo proceso, subsistente en sí mismo, en donde los procesos tuvieran lugar y en donde además éstos pudieran estar ordenados. Es necesario advertir. de otro lado, que el aceptar un tiempo subsistente en sí mismo conduce a sęudoproblemas que, como tales, resultan insolubles, por ejemplo: la negación del tiempo mismo, al pensar en el presente como división entre futuro y pasado y en éstos como no existentes, necesitándolo, no obstante, como condición para la ocurrencia de las procesos. O conduce a situaciones en las que pareciera posible pensar en propiedades del tiempo al margen de lo que ocurre en los procesos, por ejemplo: que es homogéneo, es

(2) Esta manera de entender los procesos puede encontrarse en diversos autores, por ejemplo: Mario Bunge. La investigación cientifica. Barcelona, Ediciones Ariel, 1972, p. 718. Asimismo, G. Bergman. Filosofía de la ciencia. Madrid, Tecnos, 1961, p. 110 . Igualmente, H. Reinchenbach. El sentido del tiempo. México, Universidad Nacional Autónoma de México, 1959, p. 50. 
ordenado, tiene una dirección; o por último, y mucho peor aún, puede inducirnos a pensar en un tiempo vacío (3).

Para evitar el surgimiento de semejantes dificultades resulta más adecuado pensar en los procesos como concatenaciones cualesquiera de acaecimientos. Lo único eliminado en la idea habitual es la suposición de que los procesos se den en el tiempo. Descartado tal supuesto, se advierte prontamente que sólo quien pensara en un tiempo en sí y procesos en él dados podría encontrarse con la dificultad de la negación del tiempo, justo por ser ese tiempo el concebido como constituido por pasado, presente y futuro (por lo demás, tales nociones están íntimamente ligadas a nuestros recuerdos y expectativas; y esto, aunque constituye un problema interesante, no es preciso discutirlo aquí) (4). De otro lado, resulta obvio que si tiempo es un término que designa la temporalidad y ésta sólo se da como nota constitutiva del proceso carece de sentido pensar en un tiempo vacío, es decir una temporalidad sin procesos.

Pensar que el tiempo tenga propiedades, tales como orden o dirección, es algo vinculado directamente con afirmaciones como las siguientes: hay diferencia clara entre pasado (antes) y futuro (después), el tiempo fluye de pasado a futuro, el pasado jamás retorna.

En una primera aproximación, el análisis parece mostrar que los problemas relacionados con las ideas de orden y dirección temporales están planteados en los términos de una concepción del tiempo como subsistente en sí mismo, pues sólo en ella nos parece posible considerarcaletempolcomot unStluir o poseyendo determinadas propiegdgdes. Pecifrel modo pesse pensaría en propiedades del tiempo, sino, tal vez, en caracteres de los procesos mismos, lo cual haría más claros y simples ciertos análisis, además de cambiar el terreno de la discusión. Considerada la situación de acuerdo con lo anteriormente examinado, estaríamos, seguramente, en condiciones de afirmar que los mentados problemas no deberían en verdad surgir.

Existen, no obstante, numerosas ideas asociadas a los conceptos de orden y dirección del tiempo que no parecen ser tan fácilmente eliminables, además tales nociones bien pueden estar vinculadas con los procesos mismos y no solamente con una presunta temporalidad en sí. Esto justifica, indudablemente, un tratamiento más detenido de la situación.

(3) Cf. N. Hartmann. Ontología. México, Fondo de Cultura Económica, 1960 , t. IV, p. 170 y ss.

(4) Un análisis de algunos aspectos de este problema puede encontrarse, por ejemplo, en A. Ayer. El problema del conocimiento. Buenos Aires, Editorial Universitaria de Buenos Aires, 1962, p. 177 y ss. 
Se ha considerado la posibilidad de esclarecer la noción de orden temporal reduciéndola al orden de las conexiones causales, y un buen ejemplo de esta orientación (5) se encuentra en Reichenbach quien afirma que "si definimos el orden del tiempo en función de la conexión causal, habremos mostrado cuáles son los rasgos distintivos de la realidad física que se reflejan en la estructura del tiempo y, también, habremos dado una explicación del vago concepto del orden del tiempo" (6). Por lo demás, en el mismo autor nos encontramos con la idea de que aunque la conexión puede proporcionarnos una definición del orden temporal, no permite determinar una sola dirección en el tiempo. La dirección única quedará determinada en virtud de una característica de ciertos procesos, ésta es el aumento de la entropía (7). Los procesos mecónicos, se dice, no proporcionan información respecto de la dirección del tiempo, esto por el hecho de ser considerados reversibles. De los otros procesos, los termodinámicos, que son justamente los que muestran aumento en la entropía, considerándoselos por tanto irreversibles, se afirma que, precisamente en su condición de irreversibles, nos permiten establecer una dirección única en el tiempo.

Planteado en estos términos el problema, hay en éste tres aspectos que podemos examinar. El primero se vincula con la pretendida definición del orden temporal en función de la conexión causal. El segundo está relacionado con la distinción entre orden y dirección en el tiempo. El tercero comprende la idea misma de dirección en el tiempo. En lo que sigue analizaremos únicamente los aspectos primero ye segundo. Laldiscusión acerca de si es - no correcto hablar de una direeción en el tiempo será dejada para otra oportunidad.

Veamos el modo en que puede aclararse el punto pertinente al orden temporal y la conexión causal y la definición del primero en función de la segunda.

Ocurre que si los procesos se toman como acaecimientos causalmente conectados y estas conexiones son de clase tal que algunos acaecimientos son causas y otros son efectos, se tendrá que considerar causas como anteriores y efectos como posteriores, pero entonces resulta claro que la conexión causal misma está siendo definida en función del tiempo. De otro modo no sería comprensible verdaderamente lo que quiere decirse cuando se ha-

(5) Esta idea se encuentra ya en Leibnitz; puede verse el ensayo de H. Reichenbach, titulado: "La teoría del movimiento según Newton, Leibnitz y Huyghens", en Moderna filosofía de la ciencia. Madrid, Tec-
nos, 1965.

(6) H. Reichenbach. El sentido del tiempo, p. 42 y ss.

(7) En verdad todo proceso real implica un aumento en la entropía dentro de un sistema. 
bla de causas y efectos. No puede ser de otro modo pues si la definición del orden temporal requiere que en todo caso quede determinado un "antes" y un "después", la conexión causal deberá incluir estos elementos, si es que de algún modo el orden causal representa rasgos de la realidad física que se reflejan en la estructura del tiempo.

De lo que antecede se infiere que, en cuanto a la definición respecta, se está considerando la relación causal de manera tal que si con ella se vinculan dos acaecimientos, digamos a modo de ejemplo que el acaecimiento $A$ (causa) acarrea el acaecimiento $B$ (efecto), la conexión esté sometida, por lo menos, a las siguientes condiciones: primero, que si A acarrea B, lo inverso no pueda ser sostenido como verdadero (por lo menos no en el mismo instante y en el mismo sentido); segunda, que se dé un lapso temporal entre A y B ( $A$ es anterior y B posterior).

Vistas así las cosas es de advertir que lo predicado de la conexión causal será lo previamente predicado del tiempo. Si en tal condición el orden temporal va a ser definido por el orden causal, en semejante definición no diremos más que lo que ya se hubiera dicho del tiempo. Por lo tanto, no consideramos correcto intentar definir orden causal en función de conexión causal.

Pasemos a considerar ahora el segundo aspecto del problema.

La idea de que los procesos mecánicos (concebidos como reversibles) pueden ser descritos de una manera invertida es básicamente correcta; más aún, hasta es posible pensar en encontrar procesos reales que coincidan con la descripción invertida de otros. Si tal cosa ocuiriera,-podría entonces tenerse por correcto argumentar que ciertamente los procesos mecánicos, siendo reversibles, no nos proporeibnan base suficientel para determinar una dirección única en el tiempo, pues el carácter mismo de su reversibilidad estaría indicándonos dos direcciones; imaginando, además, que el tiempo puede marchar en cualesquiera de ellas. Lo establecido sería solamente un orden temporal, pues en un proceso cualquiera y en lo que se considerara su inverso la disposición de acaecimientos sería la misma, y de este modo se encontraría siempre diferencia entre lo que es antes y lo que es después. Mas llamar a algo "antes" o "después" (pensando en un antes y un después que realmente lo fueran) dependería de la dirección en que realmente el tiempo marchara. Llegaríamos finalmente a la conclusión de que si bien los procesos reversibles permitirían establecer un orden, sería necesario examinar otro tipo de procesos si se quisiera establecer una dirección en el tiempo.

$\mathrm{Si}$ los procesos mecánicos, para los efectos de la definición del orden temporal, se interpretan como cadenas de acaecimientos causalmente conectados, de estas conexiones puede concebirse, igualmente, que son reversibles, de modo que si con ellas puede 
parecer posible definir un orden, no será, sin embargo, posible determinar una dirección.

Pero si la mentada reversibilidad es meramente ideal, no es posible ver en qué sentido son los procesos mecánicos en cuanto tales reversibles. Si la reversibilidad no es físicamente real, no es el caso entonces de que el proceso mecánico pueda mostrar dos direcciones en el tiempo; no se daría el hecho de reflejarse un rasgo de la realidad física en la estructura del tiempo. La presunta reversibilidad de los procesos mecánicos parece ser, en verdad, meramente ideal, y se da sólo como una consecuencia de las propiedades de las ecuaciones que los representan, no como manifestación de una propiedad física de tales procesos.

$\mathrm{Si}$ el orden causal presupone, como viéramos ya, un orden temporal (antes y después), resulta en apariencia razonable creer que si B está entre A y C en un "tiempo positivo", en un "tiempo negativo" (en el caso de los procesos mecánicos, nuevamente considerados reversibles) $B$ estaría entre $C$ y $A$, pues en este caso lo anterior sería posterior y viceversa, manteniéndose la diferencia entre uno y otro y por tanto un orden. Pero si lo anterior y posterior son justo eso, no parece correcto pensar sin más, que inversiones en el orden causal sean posibles, salvo el caso de proceder en el plano puramente conceptual; mas incluso en este nivel puede parecer autocontradictorio pensar que lo anterior sea posterior y viceversa mientras nos referimos al mismo proceso.

Además, que los procesos puedan ser descritos como invertidos no conduce necesariamente a pensar en dos direcciones para un orden dado en un proceso, d como sirtal orden no estableciera también una dirección. El hecho de que en las secuencias A B - C y C - B Jorge, Pebresentantes de fiempos "positivo" y "negativo" respectivamente, A sea anterior y C posterior, en un caso, y C anterior y A posterior, en otro, está, por supuesto, estableciendo una dirección para cada secuencia, y aunque, en el terreno de la abstracción, conjuntamente tomadas estas secuencias pueda pensarse en dos direcciones del tiempo, no parece razonable concluir que, por tal motivo, se esté determinando un orden solamente y no una dirección; pues, o se da como cosa de hecho, y es en este sentido que hablamos de los procesos, la secuencia $\AA-B-$ $\mathrm{C}$ y tenemos una dirección bien establecida, de $A$ hacia $C$, o se da la secuencia $C-B-A$, también con una dirección bien de terminada y única, de $C$ hacia $A, y$ no es el caso de que se den ambas secuencias conjuntamente y constituyendo un solo proceso, a no ser, claro está, que se pensara en un único y mismo proceso "caminando" hacia adelante y hacia atrás en una misma sucesión temporal, pensamiento que sería obviamente sin sentido, o por lo menos no coincidiría con ninguna de nuestras experiencias. Si se pensara que $A-B-C$ y $C-B-A$ representan dos pro- 
cesos, invertido el uno respecto del otro, puesto que en este caso se trataría de dos procesos distintos, no resultaría pertinente hablar de dos direcciones del tiempo, cada proceso constituiría una secuencia que daría lugar a un tiempo "positivo". Esto precisamente por ser la temporalidad nada más que una nota constitutiva del proceso. Cada proceso conlleva su temporalidad y a ésta podemos llamarla "positiva" en cada caso. "Positiva" en el sentido de darse efectivamente en los procesos. No se piense por tanto, erróneamente, que la temporalidad del proceso sería "positiva" únicamente respecto de una "negativa"; esto último, en nuestra opinión, carece de sentido por cuanto la idea de inversiones en el orden temporal y causal, o de tiempos "positivo" y "negativo", sólo puede surgir si se presupone deliberada 0 indeliberadamente un tiempo subsistente en sí mismo, en el cual se den los procesos y en donde éstos puedan desarrollarse hacia adelante o hacia atrás.

Visto el carácter sumamente problemático de la supuesta reversibilidad temporal y de la separación radical de orden y dirección temporales, sostenemos que es más correcto pensar, como adelantáramos ya (véase la condición primera respecto de la conexión causal), que el orden causal es unidireccional; es decir que no sería el caso de no quedar mediante éste determinada una dirección en el tiempo (si semejante cosa hubiera de admitirse, por supuesto). De esta manera puede comprenderse sin resultarnos en absoluto extraño que si se toma la conexión causal como base para construir una red causal, ésta no sólo muestre un orden, sino también una dirección, apareciendo ante nosotros como abierta. Todo esto justifica afirmaciones ecomodon dlei quenelepasado no retorna, lo que, naturalmente, coincide plenamente con la experiencia (8). Ocurre así dado que, como anteriormente mostráramos, lo que se dice de la conexión causal es nada más lo dicho respecto del tiempo, y si a éste lo pensáramos ordenado y direccional lo mismo habríamos de pensar de aquélla.

Una atenta consideración de lo expuesto nos lleva a recona cer que es el par mismo anterior-posterior, como cosa de hecho, unidireccional, y si sobre esta basse establecemos conexiones causales, éstas deberán ser igualmente undireccionales. De acuerdo con esto podemos afirmar que es la relación anterior-posterior la que nos lleva a concebir cosas como orden o dirección, y sea que de ella hablemos como de un orden o como de una dirección, realmente, en el fondo, estamos hablando de lo mismo; por tanto, no parece ya muy adecuado pretender establecer una separación en-

(8) Que la red sea abierta o cerrada es en todo caso algo que en su determinación depende de la experiencia. 
tre ambos, como si en verdad representaran situaciones diferentes. En cuanto se diga haber establecido un orden, en estos términos, se habrá establecido una dirección y viceversa. Lo expresado puede quedar más claro si tomamos como punto de partida el mundo concebido como conjunto de procesos. Sobre esta base resulta correcto pensar que en cuanto la conexión causal presupone un anterior y un posterior (causa y efecto) y los conceptos de orden y dirección temporales mencionan justamente lo mismo, tanto orden y dirección temporales como conexión causal remiten en última instancia al mismo hecho, a ese modo de ser del proceso al que denomináramos temporalidad, siendo en los procesos donde un acaecimiento puede ser considerado anterior $\mathrm{y}$ otro posterior $\mathrm{y}$ en donde éste puede llamarse efecto y aquél causa.

Así considerada la situación, deberemos reconocer que, en definitiva, no es correcto hablar de orden temporal sin admitir que éste implique necesariamente una dirección. Se confirma además la insuficiencia e inutilidad del intento de definir orden temporal en función del orden causal.

Llegados a este punto, sostenemos que es posible y enteramente correcto pensar en el orden temporal, por no hablar más que de él, como la disposición misma de los acaecimientos que constituyen los procesos, y que sea cual fuere la manera en qu€ éstos se desarrollaran siempre podríamos referirnos a ella como a un orden; pero éste sería el orden de los procesos, y no vendría al caso considerarlo, sin más, como el orden del tiempo. Si aquél es cosa de hecho, no parece pertinente tratar de definirlo de algún modo; dabe, tal vez, únicamente mostrarlo o describirlo.

Queda, no obstante ựcin última instancia, la noción de una ordenación o dirección en los procesos mismos. Determinar si hay algún motivo justificado para referirnos a los procesos en estos términos, o si estos tienen como cosa de hecho una disposición cualquiera a la que llamamos orden o dirección y nada más, o si tal vez, en relación con la posibilidad anteriormente mencionada, no tiene sentido aplicar los conceptos de orden o dirección a co. sas de hecho, es problema que esperamos examinar en otra oportunidad. 